\title{
HIRA directly targets the enhancers of selected cardiac transcription factors during in vitro differentiation of mouse embryonic stem cells
}

\author{
Rasha Noureldin M. Saleh ${ }^{1,2} \cdot$ Daniel Dilg $^{1}$ - Abla A. Abou Zeid ${ }^{2} \cdot$ Doaa I. Hashad $^{2} \cdot$ Peter J. Scambler $^{1}$. \\ Ariane L. A. Chapgier ${ }^{1}$ (D)
}

Received: 9 May 2018 / Accepted: 8 July 2018 / Published online: 20 July 2018

(c) The Author(s) 2018

\begin{abstract}
HIRA is a histone chaperone known to modulate gene expression through the deposition of H3.3. Conditional knockout of Hira in embryonic mouse hearts leads to cardiac septal defects. Loss of function mutation in HIRA, together with other chromatin modifiers, was found in patients with congenital heart diseases. However, the effects of HIRA on gene expression at earlier stages of cardiogenic mesoderm differentiation have not yet been studied. Differentiation of mouse embryonic stem cells (mESCs) towards cardiomyocytes mimics some of these early events and is an accepted model of these early stages. We performed RNA-Seq and H3.3-HA ChIP-seq on both WT and Hira-null mESCs and early cardiomyocyte progenitors of both genotypes. Analysis of RNA-seq data showed differential down regulation of cardiovascular development-related genes in Hira-null cardiomyocytes compared to WT cardiomyocytes. We found HIRA-dependent H3.3 deposition at these genes. In particular, we observed that HIRA influenced directly the expression of the transcription factors Gata6, Meis 1 and Tbx2, essential for cardiac septation, through H3.3 deposition. We therefore identified new direct targets of HIRA during cardiac differentiation.
\end{abstract}

Keywords HIRA $\cdot$ H3.3 $\cdot$ Heart $\cdot$ Cardiomyocytes $\cdot$ Embryonic stem cells $\cdot$ Differentiation $\cdot$ Enhancers

\section{Introduction}

Heart development is a complex process involving cardiogenic mesodermal differentiation into endocardium and myocardium. The myocardium is generated sequentially

Peter J. Scambler

p.scambler@ucl.ac.uk

$\triangle$ Ariane L. A. Chapgier

a.chapgier@ucl.ac.uk

Rasha Noureldin M. Saleh

r.saleh@ucl.ac.uk

Daniel Dilg

Daniel.Dilg@unige.ch

Abla A. Abou Zeid

Abla.Zeid@alexmed.adu.eg

Doaa I. Hashad

Doaa.Hashad@alexmed.edu.eg

1 Developmental Biology of Birth Defects Section, Institute of Child Health, University College London, London, UK

2 Clinical Pathology Department, Faculty of Medicine, Alexandria University, Alexandria, Egypt from cardiac precursors, primitive cardiomyocytes and definitive cardiomyocytes [1]. It requires a tightly controlled temporal gene expression pattern, which if disturbed can lead to the development of congenital heart diseases [2]. For example, mutations in Gata4 or Tbx2, important cardiac transcription factors, are associated with endocardial cushion and cardiac septal defects [3, 4]. Chromatin modifications and histone variants are essential for lineage commitment and cell fate [5, 6]. A distinct chromatin pattern specific for each stage of stem cell differentiation into cardiomyocytes was reported [7]. The histone variant H3.3 plays a role in early embryonic development [8] and lineage commitment of stem cells [9-11]. HIRA is required for genome wide enrichment of the histone variant $\mathrm{H} 3.3$ at active and bivalent genes in mouse embryonic stem cells (mESCs) [11].

HIRA is part of one of the two complexes that deposits $\mathrm{H} 3.3$ in a replication independent manner $[12,13]$. The HIRA complex includes UBN1, CABIN1 and ASF1 [14]. It deposits $\mathrm{H} 3.3$ at the gene bodies of developmentally regulated genes [11], highly active genes [10] and some active enhancers and regulatory elements [15]. It has also been shown that there is HIRA-independent H3.3 enrichment 
at certain transcription factor binding sites (TFBS) and at telomeres in undifferentiated mESCs in a ATRX/DAXX dependent manner [10].

Absence of HIRA in mice [16] and Xenopus laevis [17] leads to gastrulation defects and embryonic lethality. Attenuation of Hira expression in chick cardiac neural crest results in common arterial trunk, suggesting a role for HIRA in outflow tract (OFT) septation [18]. Hira-null mouse embryos showed abnormal heart looping and pericardial oedema at embryonic day (E)10.5 [16]. Recently, HIRA was found to be one of 46 chromatin modifiers that had a significant loss of function mutation ( $p$ value $=0.03$ ) in a large congenital heart disease cohort study [19]. We previously published that conditional knockout of Hira in the cardiogenic mesoderm resulted in embryonic lethality with atrial and ventricular septal defects at E15.5. Following genome-wide analysis of the transcriptome of these conditional knockout mouse hearts at E12.5, we identified a dysregulation of certain cardiac genes such as Tnni2, Tnnt3 and Epha3 [20]. We showed HIRA-dependent H3.3 deposition at the enhancer Tte that regulates the expression of Tnni2 and Tnnt 3 during cardiac development.

However, the role of HIRA at the early stages of cardiogenic mesoderm differentiation has not been studied. Differentiation of mESCs towards cardiomyocytes mimics the early stages of cardiogenic mesodermal differentiation [7, 21]. Here, we used in vitro mESC differentiation to study the role of HIRA during this process and focused on day 15, when the cardiogenic mesoderm genes are expressed [22]. High-throughput RNA sequencing (RNA-seq) and H3.3-HA chromatin immunoprecipitation followed by massive parallel sequencing (ChIP-seq) were then performed on both WT and Hira-null undifferentiated and mESC-derived cardiomyocytes at day 15 of differentiation. We report here that HIRA is required for the expression of cardiac transcription factors involved in septation during cardiac development. These transcription factors displayed diminished H3.3 enrichments in their gene bodies or near their Transcription Start Site (TSS) in the absence of HIRA. This reflects the requirement for HIRA in the expression of important cardiac transcription factors through H3.3 deposition.

\section{Methods}

\section{Cell culture and differentiation}

H3.3-HA tagged wild type (W9.5) and Hira-null (Clone 104) mESCs have been cultured and maintained as described previously [22]. These cells were maintained in an undifferentiated state at $37{ }^{\circ} \mathrm{C}$ and $5 \% \mathrm{CO}_{2}$ on $0.1 \%$ gelatin coated flasks in Knockout ${ }^{\mathrm{TM}} \mathrm{D}-\mathrm{MEM}$ (GIBCO, 10829), supplemented with 15\% ES-FCS (Millipore ES-009B), 1×
Glutamax (GIBCO 35050-038), 1× Penicillin/Streptomycin (GIBCO 15140), 1× MEM NEAA (GIBCO 11140-035), $0.1 \mathrm{mM} 2-\beta$-mercaptoethanol (SIGMA M-7522) and $10^{3}$ Units/ml LIF (Millipore, ESG-1106).

Differentiation was adapted from the previously described hanging drop method, with some modifications $[22,23]$. Briefly, cells were cultured in a medium made of DMEM (GIBCO 61965-026), with 15\% ES-FCS (Millipore ES-009B), 1× Penicillin/Streptomycin (GIBCO 15140), $1 \times$ MEM NEAA (GIBCO 11140-035) and $0.1 \mathrm{mM}$ $\beta$-mercaptoethanol (SIGMA M-7522). Embryoid bodies (EBs) were formed by hanging the cells in the form of drops on the lids of petri dishes at a concentration of 25 cell/ $\mu l$. After 2 days, these EBs were dropped into non-TC treated petri dishes and were grown in suspension culture for another 2 days. At day 4 , the EBs were plated in $0.1 \%$ gelatin coated tissue culture dishes and the medium was changed every day till day 15 .

\section{RNA extraction and sequencing}

RNA was extracted from two different biological samples of both WT and Hira-null cells at day 0 and day 15 using TRIzol reagent (Life technologies 15596-018) following the manufacturer's instructions. The quality and quantity of RNA were determined using Nanodrop spectrophotometer ND-1000 (Lab tech) and using High Sensitivity RNA screenTape ${ }^{\circledR}$, samples with RIN of seven or more were used for subsequent library preparation. Library preparation was done using KAPA stranded mRNA-seq kit (KAPABIOSYSTEMS KK8421) and KAPA mRNA capture kit (KAPABIOSYSTEMS KK8441). RNA sequencing was performed by Illumina NextSeq 500, with the production of paired-end reads. Reads were aligned to mouse genome $\mathrm{mm} 9$ using bowtie [24] and differential expression was processed using Deseq package version 1.6.3. The produced gene lists were sorted using an adjusted $p$ value $\leq 0.05$ and an absolute fold change of \pm 2 . Gene Ontology analysis was done using DAVID bioinformatics functional annotation tool [25]. Enrichment analysis was performed using gene set enrichment analysis (GSEA) software and the candidate genes were selected using the GSEA lead Edge tool [26]. Functional enrichment was visualized using Cytoscape to produce enrichment maps [27].

\section{Reverse transcription and quantitative real time PCR}

The High-Capacity RNA-to-cDNA ${ }^{\mathrm{TM}}$ Kit (Thermo fisher 4387406) was used to obtain cDNA and was used according to the manufacturer's instructions. Primers for qRTPCR were designed using the primer-blast tool of NCBI. The amplicon size was set between 70 and $200 \mathrm{bp}(\mathrm{bp}$ ). 
Quantitative real time PCR (qRT-PCR) was performed on the CFX96 Touch $^{\mathrm{TM}}$ Real-Time PCR Detection System using SYBR green (BIO-RAD 1708882) on three biological replicates and analysis was done using the standard curve method. Results were normalized to Gapdh.

\section{Native ChIP followed by qPCR}

Native ChIP was performed on $1 \times 10^{7}$ cells as previously described [10, 11]. Briefly, the cell pellets were lysed and chromatin was digested using MNase (Sigma N5386) at $37{ }^{\circ} \mathrm{C}$ with an ideal fragmentation between 200 and $1000 \mathrm{bp}$. The reaction was stopped using $5 \mathrm{mM}$ EDTA. HA antibody was collected with dynabeads (Invitrogen, 112.03D, 112.01D) from 12AC5 hybridoma supernatants and then incubated with the lysates overnight at $4{ }^{\circ} \mathrm{C}$. After several washes, the samples were treated with proteinase $\mathrm{K}$ and RNAse. DNA was purified using the PCR-purification Qiagen kit (28104). Quantitative real time PCR following ChIP (q-ChIP) or ChIP-seq were performed.

Primers for validation of ChIP-seq by q-ChIP were designed as follow; H3.3 significant peaks were uploaded in Integrative Genome browser (IGV). H3.3 enriched and depleted regions were identified. The corresponding nucleotide sequences were then extracted and used for primer design using NCBI primer design tool (https://www.ncbi. nlm.nih.gov/tools/primer-blast/).

\section{ChIPseq and data analysis}

The eluted DNA from both Input and ChIP was processed through library preparation using the NEB DNA Ultra kit and selecting fragment sizes of around $200 \mathrm{bp}$. Samples were sequenced using Illumina NextSeq 500, with the production of paired-end reads. Reads were aligned to mouse genome $\mathrm{mm} 9$ using BOWTIE 1.1.2 allowing no more than three mismatches [28]. Samtools 1.3.1 was used to generate BAM files, remove duplicates, sort and index [29]. MACS 1.4.2 was used for peak calling using the default parameters $\left(p\right.$ value $\leq 10^{-5}$ ) [30]. BEDTOOLS intersect with $(-v)$ option was used to generate the HIRA-dependent H3.3 peaks, after excluding the peaks in the Hira-null sample [31]. Gene lists were generated within $+10 \mathrm{~kb}$ of the TSS, in the gene body and $200 \mathrm{bp}$ dowstream TES using the PAPST (Peak Assignment and Profile Search Tool) tool [32]. Genome-wide distribution of the H3.3 ChIP-seq peaks was analyzed from both bed and wig files using the cis regulatory element annotation tools (CEAS) software [33]. The HIRA-dependent H3.3 peaks resulted from the subtraction of the Hira-null peaks from the WT peaks using bedtools.

\section{Results}

\section{Cardiac markers are dysregulated in the absence of HIRA during early cardiomyocyte differentiation}

WT and Hira-null mESCs were differentiated towards a cardiac mesodermal lineage using an adapted hanging drop protocol to increase the cardiomyocyte yield [22, 23]. Beating foci were present from day 8 of differentiation in both WT and Hira-null cells. These foci increased gradually in size during differentiation until reaching $60-70 \%$ confluence at day 15 . These beating areas arose concomitantly with the expression of the cardiac transcription factor $N k x 2.5$ at day 8 [20]. We confirmed the expression of Gata4, Mef2c, Myh6 and Myh7, as cardiac specific marker at day 15 in both WT and Hira-null cells, and the expression of the pluripotency markers Nanog and Pou $5 \mathrm{fl}$ at day 0, by qRT-PCR (Fig. 1a). We then sequenced the total RNA in undifferentiated and in differentiated WT and Hira-null cells at day 15 . We compared the expressed genes observed in our experiment at day 15 with the dataset previously published by Wamstad et al. at the cardiomyocyte (CM) stage. We found that $68.6 \%$ and $67.4 \%$ of the expressed genes in WT and Hira-null dataset respectively overlap with the reported CM stage datasets, indicating that loss of HIRA does not prevent general cardiogenic differentiation.

Gene ontology (GO) biological process analysis was performed on the genes expressed in WT and Hira-null differentiated cardiomyocytes. Genes related to heart development and morphogenesis were expressed in both cell types, indicating successful differentiation into cardiac lineage in both cells (Fig. 1b). However, GO analysis of the differentially expressed genes (DEGs) show that genes related to cardiovascular development were relatively depleted in Hira-null compared to WT mESCsdifferentiated cardiomyocytes $\left(p\right.$ value $\left.=4.1 \times 10^{-21}\right)$. On the contrary genes related to the nervous system development were upregulated in the absence of HIRA. We found 1680 DEGs of which 1641 encode known genes including 108 cardiac genes and 39 were non-coding RNAs ( $p$ value $\leq 0.05$ and $F C= \pm 2$ ) (Fig. 1c).

Gene Set Enrichment Analysis (GSEA) was performed on the DEGs and we identified 52 gene sets negatively enriched and 54 gene sets positively enriched in the absence of HIRA ( $p$ value $\leq 0.01$ and FDR $\leq 0.1$ ). Enrichment map was used to visualize and functionally categorize these different gene sets, and showed an under representation of GO-terms related to cardiovascular development, immune system development and DNA repair, and over representation of GO terms related to nervous system development and neurotransmission 


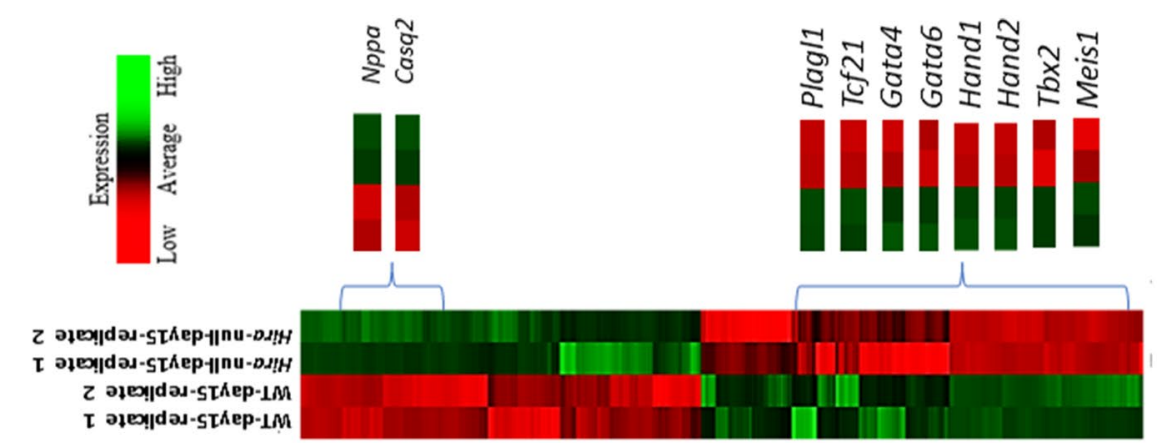

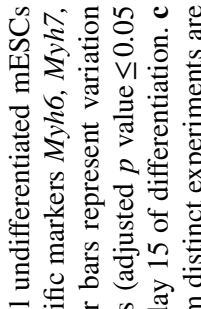

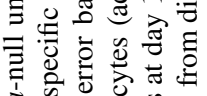

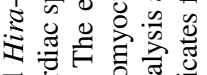

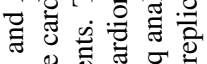

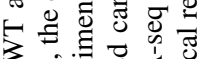

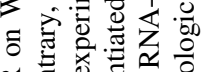

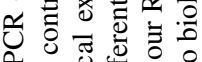

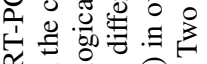

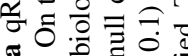

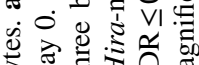

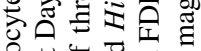

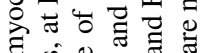

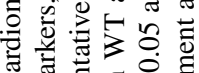

羟 $\Xi$ V 1

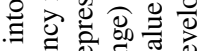

记
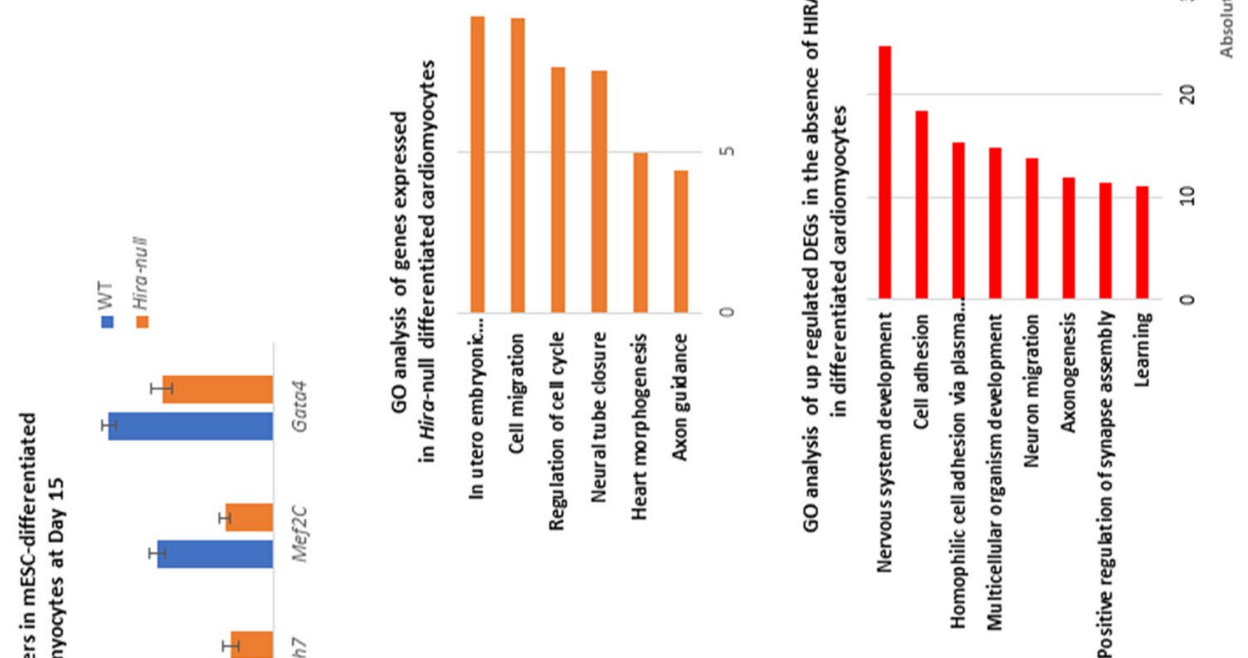

管

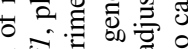

ธิ

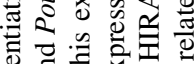

要表穸出

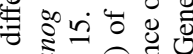

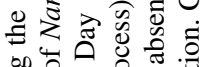

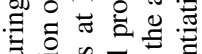

흐의

『

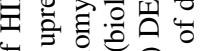

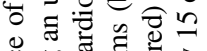

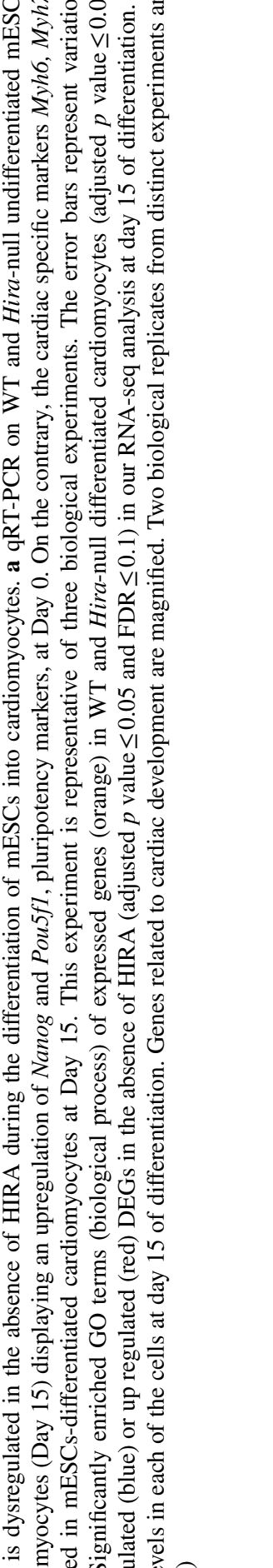

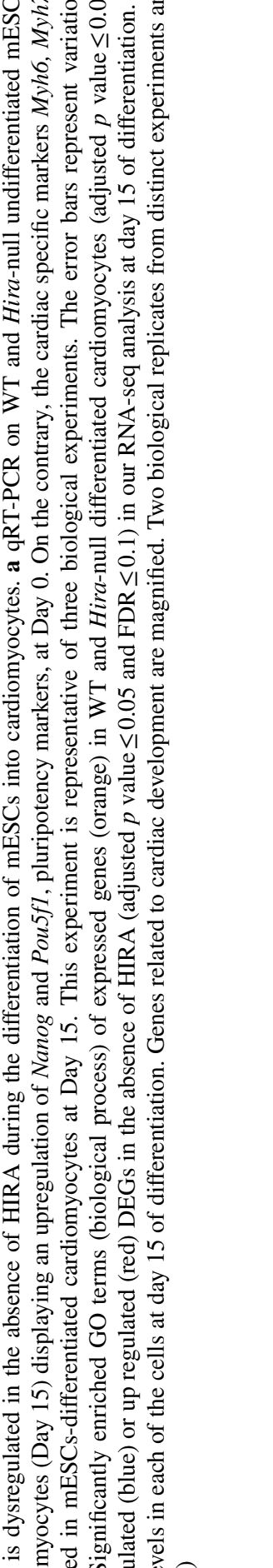

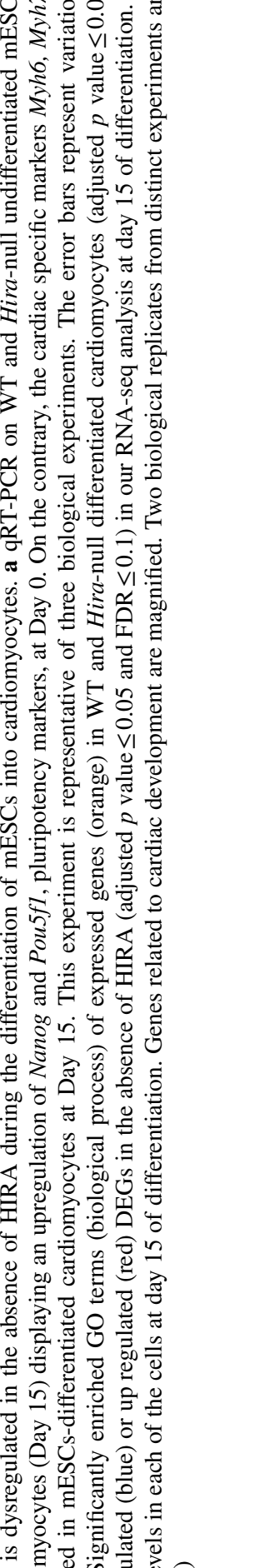

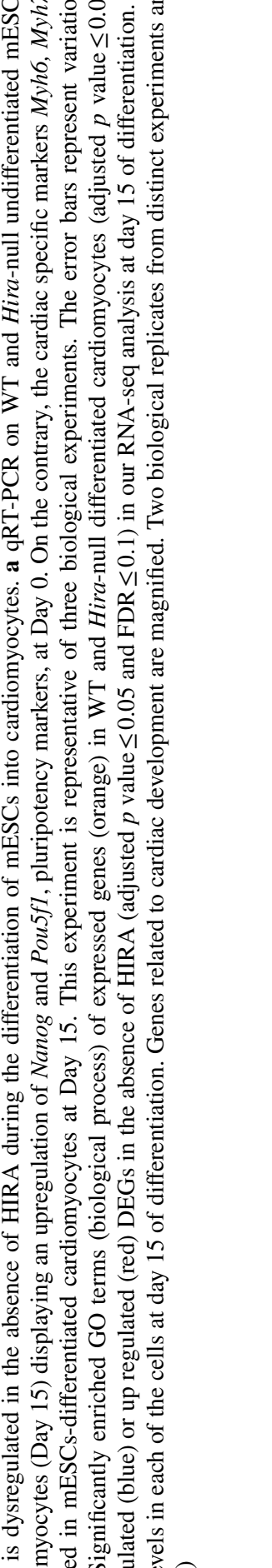

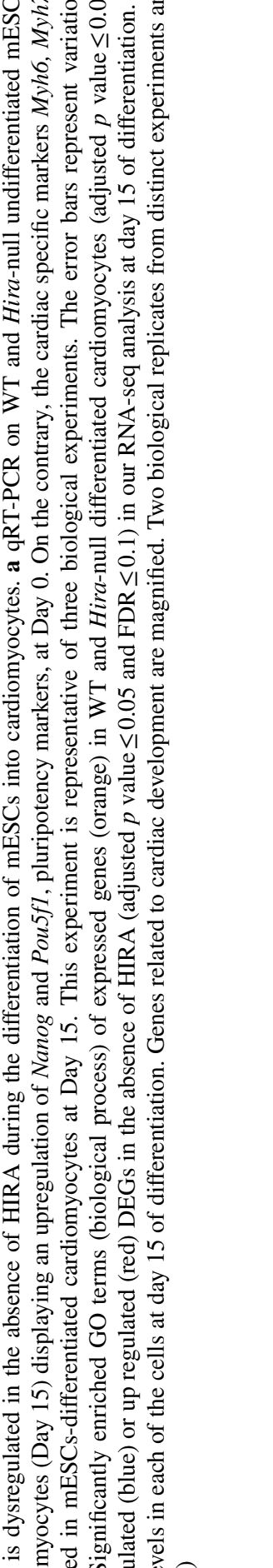

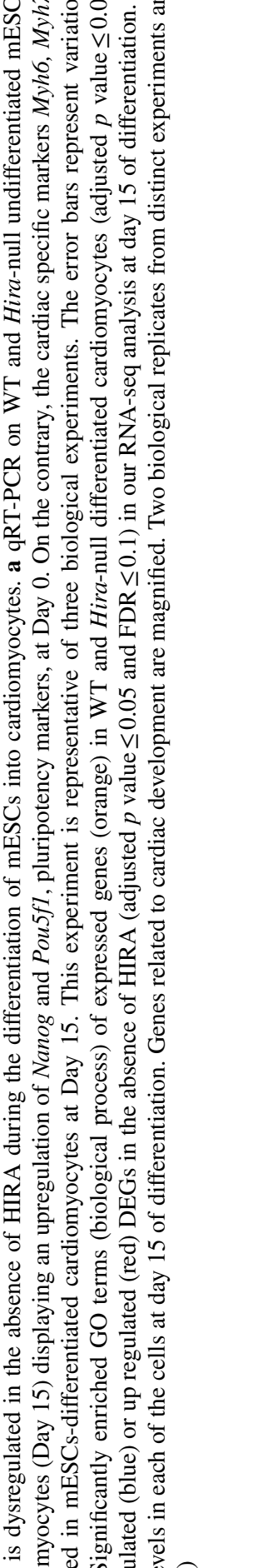

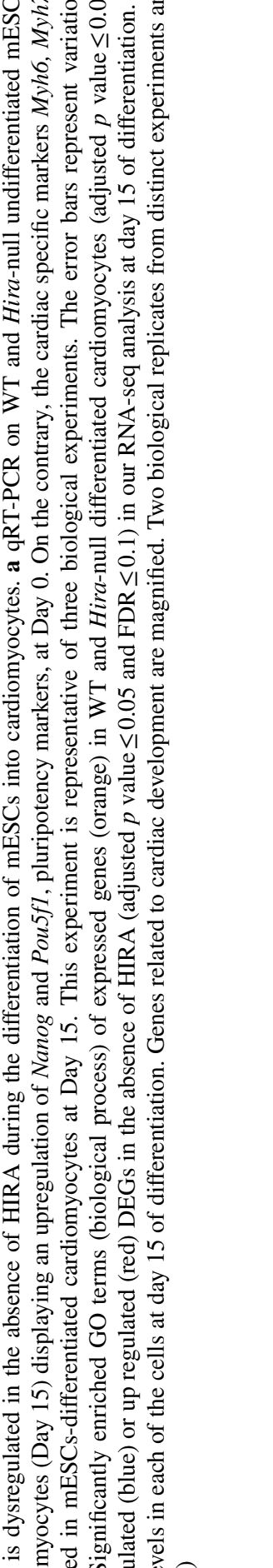

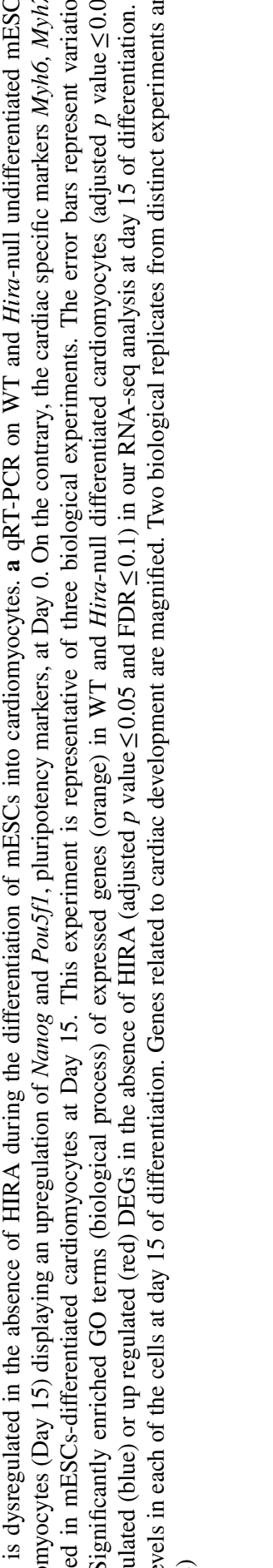
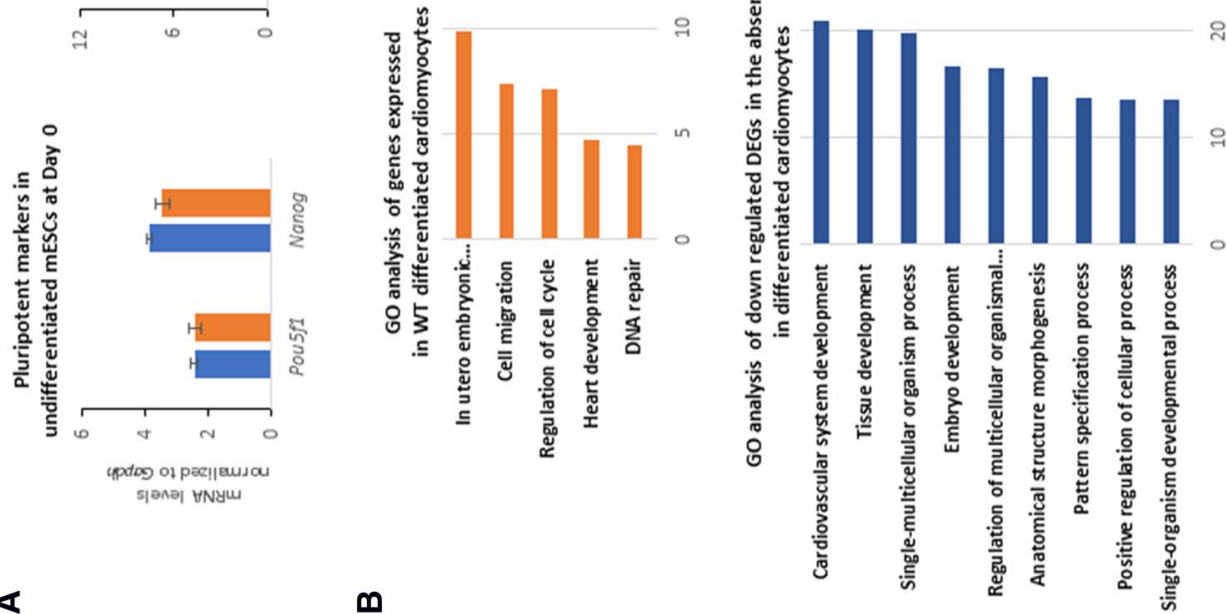

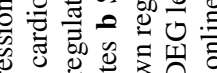

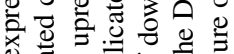

ช.

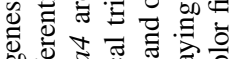

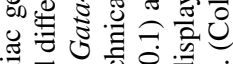

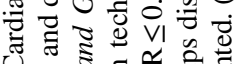

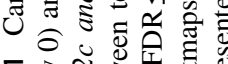

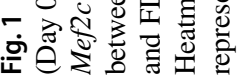



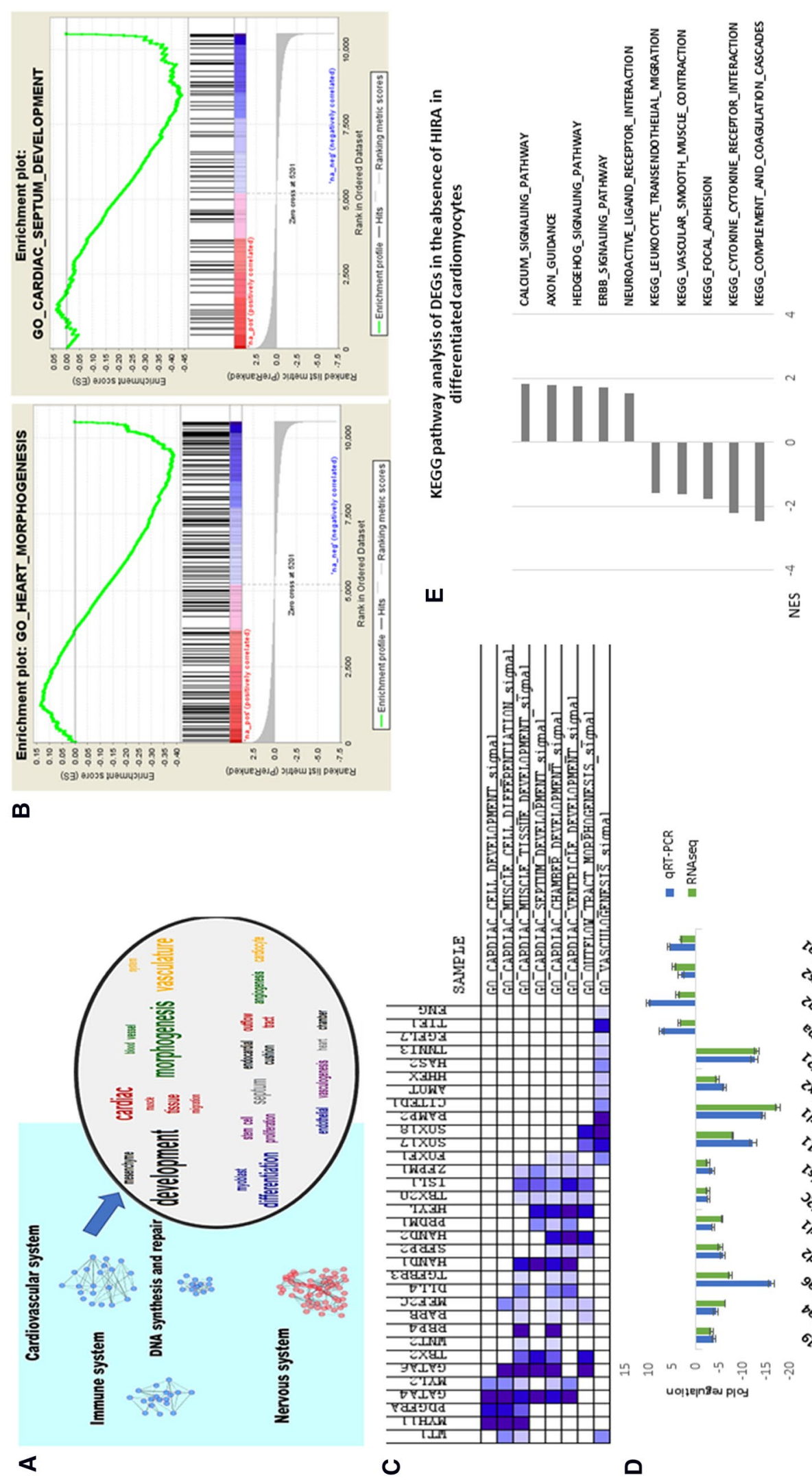

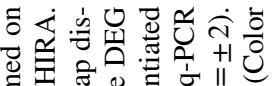

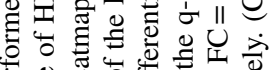

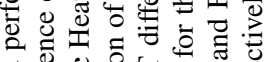
उ。ำ

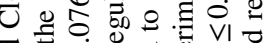

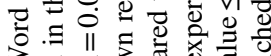

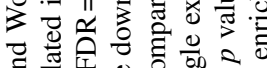

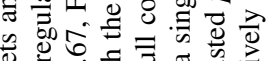

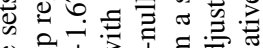

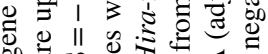

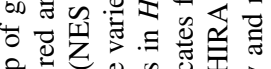

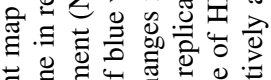

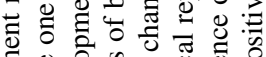

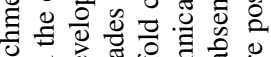
है क्षै

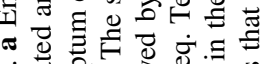

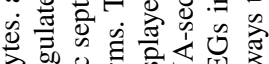

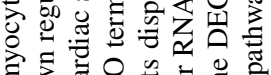
不

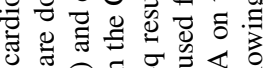
ᄋ ฮ

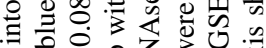

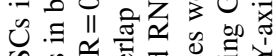

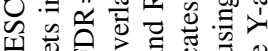

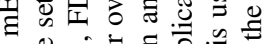
ठै

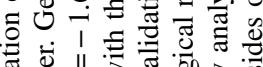

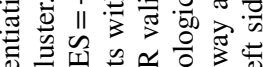

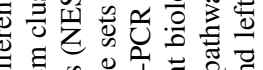
氜

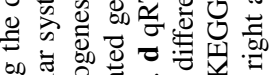

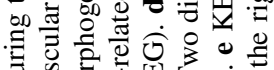

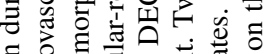

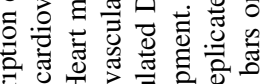

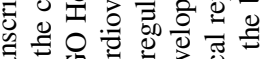

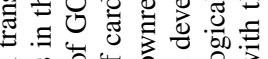
б है

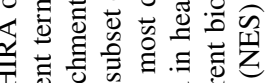

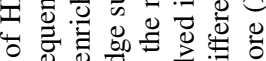

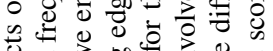

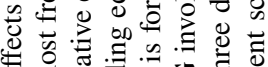
o

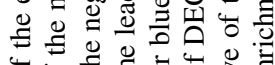

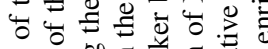

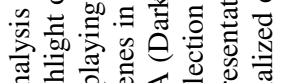

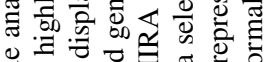

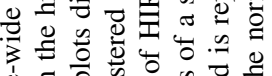

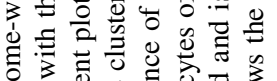

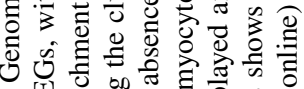

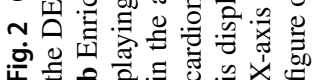


(Fig. 2a). Accordingly, the gene sets related to cardiac septum development and cardiac chamber development were both negatively enriched (Fig. 2b). We then examined the genes that were common in the different cardiovascular development gene sets using the lead edge analysis tool in GSEA. We found that Gata4, Gata6, Hand1, Hand2, Isll and $T b x 2$, and other genes represented in Fig. 2c, were common in several gene sets. We then validated a subset of these genes by qRT-PCR from independently isolated mRNA sample sets (Fig. 2d).

Altogether, the expression of cardiovascular development-related genes, and more precisely cardiac chamber and septal development associated genes was down regulated in the absence of HIRA.

\section{HIRA affects calcium signalling and vascular smooth muscle contraction pathways}

We then investigated the pathways affected by the absence of HIRA. KEGG pathway analysis was done on the pre-ranked list of DEGs using GSEA ( $p$ value $\leq 0.05$ and $\mathrm{FC}= \pm 2$ ). Interestingly, calcium signalling pathway was the most positively enriched (normalized enrichment score $(\mathrm{NES})=1.82$, $p$ value $=0.019$ and FDR $=0.19)$. In addition, axon guidance and neuroactive ligand-receptor interaction pathways, two pathways related to the nervous system, were positively enriched. Conversely, the vascular smooth muscle contraction pathway was negatively enriched (NES $=-1.63, p$ value $=0.026$ and $\mathrm{FDR}=0.17$ ). Considering the studies that link HIRA to interferon-stimulated genes [34], it is interesting to mention that leukocyte transendothelial migration pathways, which is related to the immune system is also negatively enriched (Fig. 2e).

\section{HIRA-dependent H3.3 deposition co-localizes with active enhancer loci in WT cells and shifts to distal intergenic regions in the absence of HIRA}

We previously showed that H3.3 deposition at the cardiac enhancer, Tte, is HIRA dependent [20]. However, there has been no study investigating the genome-wide effect of HIRA on the H3.3 deposition during the early stages of cardiovascular development. We therefore performed H3.3-HA ChIP-seq on undifferentiated mESCs and differentiated cardiomyocytes at day 15 that contained about $60 \%$ of beating foci and $67 \%$ of transcriptome similarly to the previously published CM stage [7].

In WT-differentiated cardiomyocytes, H3.3 was deposited most abundantly in the intronic (53.3\%) region with lesser deposition in the distal intergenic region (23.4\%), and the promotor region $(9.2 \%)$. In the absence of HIRA, the proportion of $\mathrm{H} 3.3$ deposition increased at the distal intergenic region $(42.2 \%)$ compared to the intronic region $(29 \%)$ and the promotor region (3\%) (Fig. 3a). We identified 14680 HIRA-dependent H3.3 enrichment peaks encompassing $+10 \mathrm{~kb}$ upstream of the TSS, the gene body or $200 \mathrm{bp}$ downstream of the TES of 4703 annotated genes. GO analysis showed enrichment of DNA-dependent regulation of transcription processes. We then compared these with the DEG list and found 319 in common.

Interestingly, 60 of the genes enriched for HIRA-dependent H3.3 deposition were related to heart development by GO analysis. We then compared our datasets with previously published datasets for the known enhancer marks H3K4me1 and H3K27ac in cardiomyocyte-differentiated mESCs [7]. We identified that $31.6 \%(4642 / 14,680)$ of the total HIRA-dependent H3.3 enrichment peaks overlapped with both the active enhancer histone marks $\mathrm{H} 3 \mathrm{~K} 4 \mathrm{me} 1$ and H3K27ac implicating that approximately one-third of the HIRA-dependent H3.3 deposition is at active enhancer sites (Fig. 3b).

In conclusion, the absence of HIRA leads to a decrease in the deposition of H3.3 in the intronic and promoter regions. We also show that about third of HIRA-dependent H3.3 peaks co localize with active enhancers loci.

\section{HIRA-dependent H3.3 deposition directly influences the transcription of Meis 1, Gata6 and Tbx2}

We next investigated the co-localization of HIRA-dependent H3.3 deposition with some previously published ChIPseq datasets of cardiac transcription factors (NKX2.5, GATA4, TBX5 [35] and SRF [36]). We found that 1607 of the HIRAdependent H3.3 peaks (10.93\%) overlapped with NKX2.5 peaks, 1420 peaks $(9.67 \%)$ overlapped with SRF peaks, 937 peaks (6.38\%) overlapped with GATA4 peaks and 450 peaks (3.06\%) overlapped with TBX5 peaks. Since we previously showed that HIRA and NKX2.5 bind to the common enhancer Tte [20], we focussed on identifying the colocalization of HIRA dependent H3.3 deposition and NKX2.5 at the enhancer loci and/or at the promotor site of three cardiac transcription factors Meis 1, Gata6 and Tbx2, which were downregulated in the absence of HIRA (Fig. 2d). These are known to be associated with cardiac septal defects, as we observed in our cardiac mesoderm Hira-conditional mice.

MEIS1 is a homeobox protein found to influence heart development. Mouse embryos lacking MEIS1 showed VSDs and overriding of aorta [37]. We found several HIRAdependent H3.3 enrichment sites in the gene body of Meis I (Fig. 4a). One of the most H3.3-enriched loci co-localizes with NKX2.5 at an enhancer locus marked by H3K4me1 and H3K27ac. We validated this enrichment by ChIP followed by qPCR using primers designed for this locus.

TBX2 is a member of the T-box transcription factor gene family. Absence of TBX2 leads to outflow tract septation defects [4]. We found HIRA dependent H3.3 enrichment in 
A

H3.3-HA ChIPseq

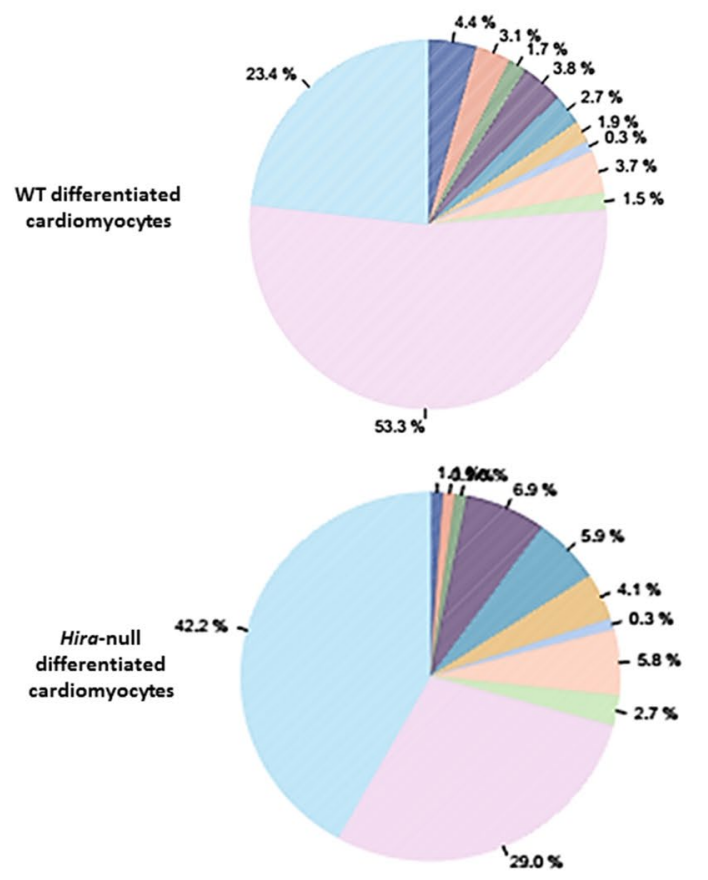

B
Fig. 3 Genome wide distribution of H3.3 in WT and Hira-null differentiated cardiomyocytes and motif analysis of the significant peaks. a Pie chart showing the distribution of $\mathrm{H} 3.3$ over different genomic regions in WT and Hira-null differentiated cardiomyocytes as indi- cated. b Venn diagram showing the overlap between HIRA-dependent H3.3 peaks and the common peaks of H3K4me1 and H3K27ac. (Color figure online)

role of the H3.3 histone chaperone HIRA in the early stages of heart development using an in vitro mESC differentiation model. We successfully differentiated both WT and Hiranull mESCs into cardiomyocytes. This was evidenced by the enrichment of heart development and morphogenesis GO terms.

We report new direct targets of HIRA-mediated H3.3 deposition, which are transcription factors known to be involved in cardiac septum formation. We previously demonstrated in vivo that mesodermal conditional Hira-null embryos presented with a fully penetrant phenotype of ventricular septal defect [20]. In that same study, we focussed on the dysregulation of expression of Tnni2 and Tnnt3, suggesting a mechanism in which HIRA with NKX2.5 binds to their common enhancer Tte to down regulate their expression. We herein predict further targets of the same mechanism: co-occupancy of HIRA and NKX2.5 at the enhancers of Meis 1 and Gata6, and at the promoter of $T b x 2$ to activate their expression. Further studies will be needed to investigate the downstream effect of HIRA on these new targets in vivo, and to explore the interactions of HIRA and NKX2.5 at these loci.

Interestingly, knockout of Meis 1 is associated with VSD and overriding aorta in mice [37], and an increased cardiomyocyte proliferation in zebrafish, suggesting a
Heart development is a complex tightly regulated process requiring strict control of temporal gene expression [40-42] and involving epigenetic regulation [43]. We examined the 


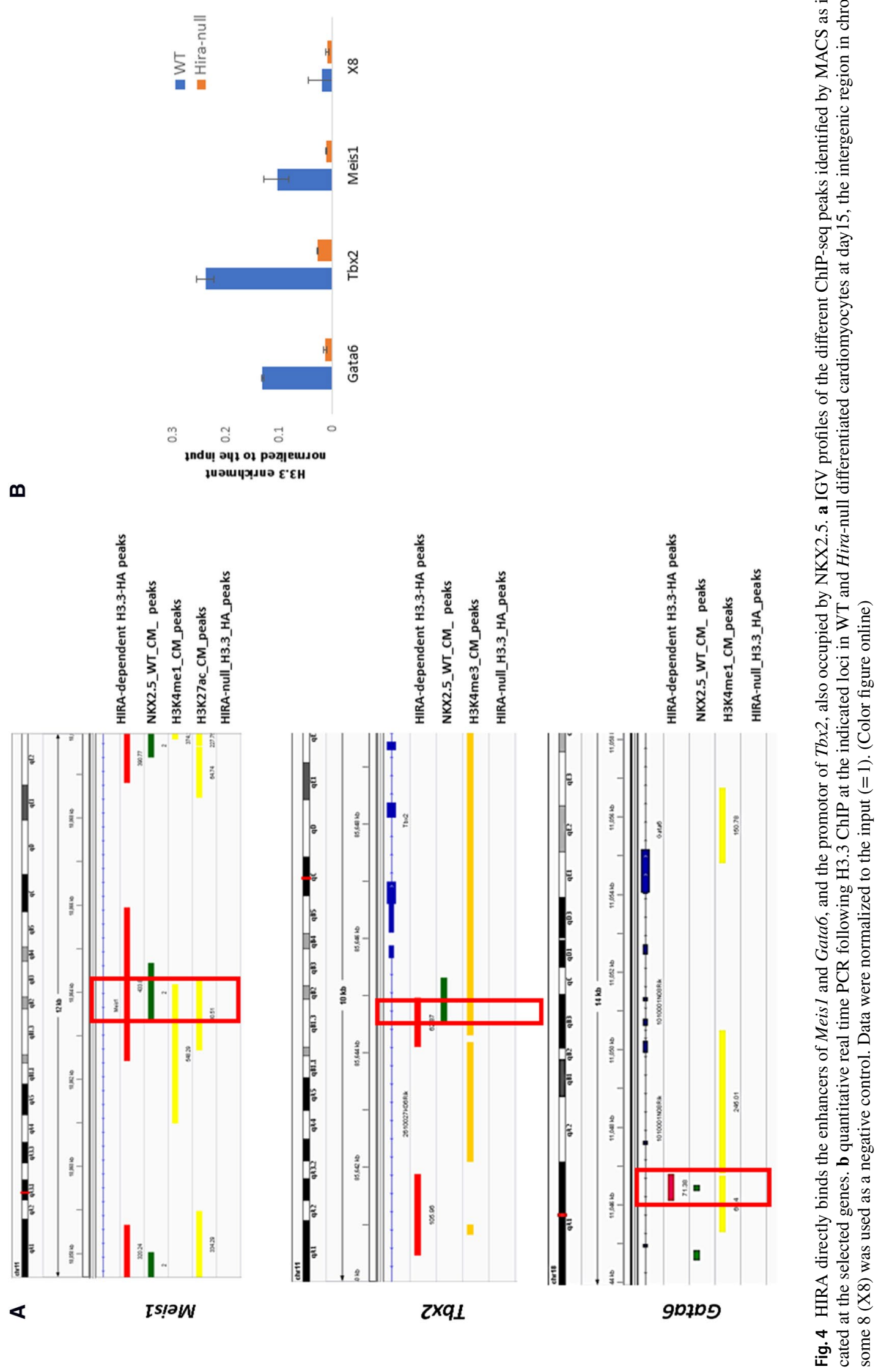


role in the cell cycle [44]. In our differentiated cardiomyocytes, Meisl was down regulated $(\mathrm{FC}=-2.5)$ in the absence of HIRA. Notably, there was co-localization of HIRA-dependent H3.3 deposition with NKX2.5 binding sites at a previously identified enhancer site. A previous study showed that the sequential binding of MEIS1 then NKX2.5 to enhancers can influence cardiogenesis [45]. Taken together with our previously published finding, which showed diminished binding of NKX2.5 at the enhancer site Tte in the absence of HIRA, we hypothesize that the absence of HIRA leads to a diminished expression of Meis 1 and then to a diminished binding of NKX2.5 to the MEIS1-NKX2.5 target sites, thereby disrupting cardiac septum morphogenesis.

Similarly, Gata6 expression was down regulated $(\mathrm{FC}=-6.1)$ in the absence of HIRA at day 15 of differentiation, and we identified HIRA-dependent H3.3, H3K4me1 and NKX2.5 enrichments co-localising at $5.5 \mathrm{~kb}$ upstream of the TSS of Gata6 in WT cells at day 15 of differentiation. It is interesting to note that the identical locus had been shown previously to be an enhancer bound by NKX2.5 and regulating Gata6 expression in developing murine hearts [39].

TBX2 plays an essential role in the atrioventricular canal and OFT development [4]. We found HIRA-dependent H3.3 deposition at the promotor of $T b x 2$ in WT cells differentiated at day 15 and a down regulation of $T b x 2$ expression ( $\mathrm{FC}=-4.6)$ in the absence of HIRA at day 15 of differentiation. This locus coincides with known NKX2.5 binding sites and a peak of the active transcription mark $\mathrm{H} 3 \mathrm{~K} 4 \mathrm{me} 3$. This result suggests that HIRA may play a role in the atrioventricular canal and the OFT development by influencing the transcription of $T b x 2$ through $\mathrm{H} 3.3$ deposition at its promotor.

In addition, we found that calcium signalling pathway was significantly upregulated in the absence of HIRA at day 15 of differentiation. Calcium signalling controls several physiological processes in the heart, including cardiac contractility [46]. KEGG calcium signalling pathway is normally required in ESC-derived cardiomyocytes [47]. We previously reported two cardiac genes involved in contractibility to be up regulated in the hearts of cardiac mesoderm Hira-conditional mice. Further investigations on cardiac contractility in Hira-mutant mice would be interesting.

Outside of heart development, it is of interest to note that in our study, gene sets involved in DNA repair, the development of the immune and nervous systems were significantly dysregulated in the absence of HIRA. Of relevance, HIRA has been shown to have an essential role in transcriptional recovery after DNA damage $[48,49]$, and several studies link HIRA-dependent H3.3 deposition with the induction of stress responsive associated genes following interferons and heat-shock stimulations $[34,50]$. Absence of HIRA in neural progenitors resulted in decreased proliferation and increased neuronal differentiation [51]. Our study provides new subsets of genes that could be investigated further in these areas.

In conclusion, we show that cardiovascular developmentrelated genes are expressed in both WT and Hira-null differentiated cardiomyocytes with a significant lower expression in Hira-null cells. We show that $31.6 \%$ of HIRA-dependent H3.3 peaks co-localize with active enhancers loci. We found that HIRA directly influence the expression of the cardiac transcription factors Meis1, Gata6 and Tbx2. We suggest that HIRA influences their expression through the deposition of H3.3 at their enhancers, supporting our previous published model that HIRA acts at enhancers during cardiovascular development, and potentially in concert with NKX2.5 [20].

Acknowledgements We thank Laura Banaszynski and David Allis for the knockin H3.3 cells and the 12AC5 hybridoma lines. We also thank Tony Brooks from UCL Genomics for carrying out RNAseq and ChIPseq, and Benjamin Lee for bioinformatics advice. This research was funded by the Egyptian Cultural affairs and Missions Section, Ministry of Higher Education (Scholarship to Rasha Noureldin Saleh), the British Heart Foundation (FS/10/031/28395 to Ariane Chapgier), the Leducq Foundation (TNE15CVD01 to Peter Scambler) and Gt. Ormond St. Children's Charity (V2318 to Peter Scambler), and supported by the NIHR Great Ormond Street Hospital Biomedical Research Centre. The views expressed are those of the author(s) and not necessarily those of the NHS, the NIHR or the Department of Health.

\section{Compliance with ethical standards}

Conflict of interest Authors declare no potential conflicts of interest.

Ethical approval This study was conducted on mouse embryonic stem cell lines. All work was carried out under the UCL Research Ethics Committee Guidelines.

Open Access This article is distributed under the terms of the Creative Commons Attribution 4.0 International License (http://creativeco mmons.org/licenses/by/4.0/), which permits unrestricted use, distribution, and reproduction in any medium, provided you give appropriate credit to the original author(s) and the source, provide a link to the Creative Commons license, and indicate if changes were made.

\section{References}

1. Murry CE, Keller G (2008) Differentiation of embryonic stem cells to clinically relevant populations: lessons from embryonic development. Cell 132(4):661-680

2. Evans SM, Yelon D, Conlon FL, Kirby ML (2010) Myocardial lineage development. Circ Res 107(12):1428-1444

3. Rajagopal SK, Ma Q, Obler D, Shen J, Manichaikul A, TomitaMitchell A et al (2007) Spectrum of heart disease associated with murine and human GATA4 mutation. J Mol Cell Cardiol 43(6):677-685

4. Harrelson Z, Kelly RG, Goldin SN, Gibson-Brown JJ, Bollag RJ, Silver LM et al (2004) Tbx2 is essential for patterning the atrioventricular canal and for morphogenesis of the outflow tract during heart development. Development 131(20):5041-5052 
5. Buschbeck M, Hake SB (2017) Variants of core histones and their roles in cell fate decisions, development and cancer. Nat Rev Mol Cell Biol 18(5):299-314

6. Sarmento OF, Digilio LC, Wang Y, Perlin J, Herr JC, Allis CD et al (2004) Dynamic alterations of specific histone modifications during early murine development. J Cell Sci 117(19):4449-4459

7. Wamstad JA, Alexander JM, Truty RM, Shrikumar A, Li F, Eilertson KE et al (2012) Dynamic and coordinated epigenetic regulation of developmental transitions in the cardiac lineage. Cell 151(1):206-220

8. Akiyama T, Suzuki O, Matsuda J, Aoki F (2011) Dynamic replacement of histone $\mathrm{H} 3$ variants reprograms epigenetic marks in early mouse embryos. PLoS Genet 7(10):e1002279

9. Song TY, Yang JH, Park JY, Song Y, Han JW, Youn HD et al (2012) The role of histone chaperones in osteoblastic differentiation of $\mathrm{C} 2 \mathrm{C} 12$ myoblasts. Biochem Biophys Res Commun 423(4):726-732

10. Goldberg AD, Banaszynski LA, Noh KM, Lewis PW, Elsaesser SJ, Stadler S et al (2010) Distinct factors control histone variant $\mathrm{H} 3.3$ localization at specific genomic regions. Cell 140(5):678-691

11. Banaszynski LA, Wen D, Dewell S, Whitcomb SJ, Lin M, Diaz $\mathrm{N}$ et al (2013) Hira-dependent histone H3.3 deposition facilitates PRC2 recruitment at developmental loci in ES cells. Cell 155(1):107-120

12. Ray-Gallet D, Woolfe A, Vassias I, Pellentz C, Lacoste N, Puri A et al (2011) Dynamics of histone $\mathrm{H} 3$ deposition in vivo reveal a nucleosome gap-filling mechanism for $\mathrm{H} 3.3$ to maintain chromatin integrity. Mol Cell 44(6):928-941

13. Elsaesser SJ, Allis CD (2010) HIRA and Daxx constitute two independent histone $\mathrm{H} 3.3$-containing predeposition complexes. Cold Spring Harb Symp Quant Biol 75:27-34

14. Banumathy G, Somaiah N, Zhang R, Tang Y, Hoffmann J, Andrake M et al (2009) Human UBN1 is an ortholog of yeast $\mathrm{Hpc} 2 \mathrm{p}$ and has an essential role in the HIRA/ASF1a chromatin-remodeling pathway in senescent cells. Mol Cell Biol 29(3):758-770

15. Jin C, Zang C, Wei G, Cui K, Peng W, Zhao K et al (2009) H3.3/ H2A.Z double variant-containing nucleosomes mark "nucleosome-free regions' of active promoters and other regulatory regions. Nat Genet 41(8):941-945

16. Roberts C, Sutherland HF, Farmer H, Kimber W, Halford S, Carey A et al (2002) Targeted mutagenesis of the Hira gene results in gastrulation defects and patterning abnormalities of mesoendodermal derivatives prior to early embryonic lethality. Mol Cell Biol 22(7):2318-2328

17. Szenker E, Lacoste N, Almouzni G (2012) A developmental requirement for HIRA-dependent H3.3 deposition revealed at gastrulation in Xenopus. Cell Rep 1(6):730-740

18. Farrell MJ, Stadt H, Wallis KT, Scambler P, Hixon RL, Wolfe R et al (1999) HIRA, a DiGeorge syndrome candidate gene, is required for cardiac outflow tract septation. Circ Res 84(2):127-135

19. Jin SC, Homsy J, Zaidi S, Lu Q, Morton S, DePalma SR et al (2017) Contribution of rare inherited and de novo variants in 2,871 congenital heart disease probands. Nat Genet 49(11):1593-1601

20. Dilg D, Saleh RN, Phelps SE, Rose Y, Dupays L, Murphy C et al (2016) HIRA Is required for heart development and directly regulates Tnni2 and Tnnt3. PLoS ONE 11(8):e0161096

21. Luna-Zurita L, Stirnimann CU, Glatt S, Kaynak BL, Thomas S, Baudin F et al (2016) Complex interdependence regulates heterotypic transcription factor distribution and coordinates cardiogenesis. Cell 164(5):999-1014

22. Fuegemann CJ, Samraj AK, Walsh S, Fleischmann BK, Jovinge S, Breitbach M (2010) Differentiation of mouse embryonic stem cells into cardiomyocytes via the hanging-drop and mass culture methods. Curr Protoc Stem Cell Biol 15:1F-11

23. Wang X, Yang P (2008) In vitro differentiation of mouse embryonic stem (mES) cells using the hanging drop method. J Vis Exp $17: 825$

24. Langmead B, Trapnell C, Pop M, Salzberg SL (2009) Ultrafast and memory-efficient alignment of short DNA sequences to the human genome. Genome Biol 10(3):R25

25. Huang da W, Sherman BT, Lempicki RA (2009) Systematic and integrative analysis of large gene lists using DAVID bioinformatics resources. Nat Protoc 4(1):44-57

26. Subramanian A, Tamayo P, Mootha VK, Mukherjee S, Ebert BL, Gillette MA et al. (2005) Gene set enrichment analysis: a knowledge-based approach for interpreting genome-wide expression profiles. Proc Natl Acad Sci 102(43):15545-15550

27. Shannon P, Markiel A, Ozier O, Baliga NS, Wang JT, Ramage D et al (2003) Cytoscape: a software environment for integrated models of biomolecular interaction networks. Genome Res 13(11):2498-2504

28. Langmead B (2010) Aligning short sequencing reads with Bowtie. Curr Protoc Bioinform 32:11-17

29. Li H, Handsaker B, Wysoker A, Fennell T, Ruan J, Homer N et al (2009) The sequence alignment/map format and SAM tools. Bioinformatics 25(16):2078-2079

30. Zhang Y, Liu T, Meyer CA, Eeckhoute J, Johnson DS, Bernstein $\mathrm{BE}$ et al (2008) Model-based analysis of ChIP-Seq (MACS). Genome Biol 9(9):R137

31. Quinlan AR, Hall IM (2010) BEDTools: a flexible suite of utilities for comparing genomic features. Bioinformatics 26(6):841-842

32. Bible PW, Kanno Y, Wei L, Brooks SR, O'Shea JJ, Morasso MI et al (2015) PAPST, a user friendly and powerful java platform for ChIP-Seq peak co-localization analysis and beyond. PLoS ONE 10(5): $\mathrm{e} 0127285$

33. Shin H, Liu T, Manrai AK, Liu XS (2009) CEAS: cis-regulatory element annotation system. Bioinformatics 25(19):2605-2606

34. Tamura T, Smith M, Kanno T, Dasenbrock H, Nishiyama A, Ozato K (2009) Inducible deposition of the histone variant H3.3 in interferon-stimulated genes. J Biol Chem 284(18):12217-12225

35. Luna-Zurita L, Stirnimann Christian U, Glatt S, Kaynak Bogac $\mathrm{L}$, Thomas S, Baudin F et al. Complex interdependence regulates heterotypic transcription factor distribution and coordinates cardiogenesis. Cell.164(5):999-1014

36. He A, Kong SW, Ma Q, Pu WT (2011) Co-occupancy by multiple cardiac transcription factors identifies transcriptional enhancers active in heart. Proc Natl Acad Sci USA 108(14):5632-5637

37. Stankunas K, Shang C, Twu KY, Kao SC, Jenkins NA, Copeland NG et al (2008) Pbx/Meis deficiencies demonstrate multigenetic origins of congenital heart disease. Circ Res 103(7):702-709

38. Laforest B, Nemer M (2011) GATA5 interacts with GATA4 and GATA6 in outflow tract development. Dev Biol 358(2):368-378

39. Molkentin JD, Antos C, Mercer B, Taigen T, Miano JM, Olson EN (2000) Direct activation of a GATA6 cardiac enhancer by Nkx2.5: evidence for a reinforcing regulatory network of Nkx2.5 and GATA transcription factors in the developing heart. Dev Biol 217(2):301-309

40. Anderson RH, Webb S, Brown NA, Lamers W, Moorman A (2003) Development of the heart: (3) formation of the ventricular outflow tracts, arterial valves, and intrapericardial arterial trunks. Heart 89(9):1110-1118

41. Anderson RH, Webb S, Brown NA, Lamers W, Moorman A (2003) Development of the heart: (2) septation of the atriums and ventricles. Heart 89(8):949-958

42. Moorman A, Webb S, Brown NA, Lamers W, Anderson RH (2003) Development of the heart: (1) formation of the cardiac chambers and arterial trunks. Heart 89(7):806-814 
43. Han P, Hang CT, Yang J, Chang CP (2011) Chromatin remodeling in cardiovascular development and physiology. Circ Res 108(3):378-396

44. Mahmoud AI, Kocabas F, Muralidhar SA, Kimura W, Koura AS, Thet $S$ et al (2013) Meis1 regulates postnatal cardiomyocyte cell cycle arrest. Nature 497(7448):249-253

45. Dupays L, Shang C, Wilson R, Kotecha S, Wood S, Towers N et al (2015) Sequential binding of MEIS1 and NKX2-5 on the Popdc2 gene: a mechanism for spatiotemporal regulation of enhancers during cardiogenesis. Cell Rep 13(1):183-195

46. Kontula K, Laitinen PJ, Lehtonen A, Toivonen L, Viitasalo M, Swan H (2005) Catecholaminergic polymorphic ventricular tachycardia: recent mechanistic insights. Cardiovasc Res 67(3):379-387

47. Doss MX, Winkler J, Chen S, Hippler-Altenburg R, Sotiriadou I, Halbach $\mathrm{M}$ et al (2007) Global transcriptome analysis of murine embryonic stem cell-derived cardiomyocytes. Genome Biol $8(4): R 56$
48. Adam S, Polo SE, Almouzni G (2013) Transcription recovery after DNA damage requires chromatin priming by the $\mathrm{H} 3.3$ histone chaperone HIRA. Cell 155(1):94-106

49. Valenzuela N, Fan Q, Fa'ak F, Soibam B, Nagandla H, Liu Y et al (2016) Cardiomyocyte-specific conditional knockout of the histone chaperone HIRA in mice results in hypertrophy, sarcolemmal damage and focal replacement fibrosis. Dis Models Mech 9(3):335-345

50. Kim H, Heo K, Choi J, Kim K, An W (2011) Histone variant H3.3 stimulates HSP70 transcription through cooperation with HP1 gamma. Nucleic Acids Res 39(19):8329-8341

51. Li Y, Jiao J (2017) Histone chaperone HIRA regulates neural progenitor cell proliferation and neurogenesis via beta-catenin. J Cell Biol 216(7):1975-1992 